\title{
Toward the Development of Iteration Procedures for the Interval-Based Simulation of Fractional-Order Systems
}

\author{
Andreas Rauh and Julia Kersten ${ }^{a}$
}

\begin{abstract}
In many fields of engineering as well as computational physics, it is necessary to describe dynamic phenomena which are characterized by an infinitely long horizon of past state values. This infinite horizon of past data then influences the evolution of future state trajectories. Such phenomena can be characterized effectively by means of fractional-order differential equations. In contrast to classical linear ordinary differential equations, linear fractional-order models have frequency domain characteristics with amplitude responses that deviate from the classical integer multiples of $\pm 20 \mathrm{~dB}$ per frequency decade and, respectively, deviate from integer multiples of $\pm \frac{\pi}{2}$ in the limit values of their corresponding phase response. Although numerous simulation approaches have been developed in recent years for the numerical evaluation of fractional-order models with point-valued initial conditions and parameters, the robustness analysis of such system representations is still a widely open area of research. This statement is especially true if interval uncertainty is considered with respect to initial states and parameters. Therefore, this paper summarizes the current state-of-the-art concerning the simulation-based analysis of fractional-order dynamics with a restriction to those approaches that can be extended to set-valued (interval) evaluations for models with bounded uncertainty. Especially, it is shown how verified simulation techniques for integer-order models with uncertain parameters can be extended toward fractional counterparts. Selected linear as well as nonlinear illustrating examples conclude this paper to visualize algorithmic properties of the suggested interval-based simulation methodology and point out directions of ongoing research.
\end{abstract}

Keywords: interval analysis, fractional-order differential equations, Picard iteration, exponential enclosure techniques, Mittag-Leffler functions

${ }^{a}$ University of Rostock, Chair of Mechatronics, Justus-von-Liebig-Weg 6, D-18059 Rostock, Germany. E-mail: Andreas.Rauh@interval-methods.de, Julia.Kersten@uni-rostock.de. ORCID: https://orcid.org/\{0000-0002-1548-6547, 0000-0002-1160-8623\}. 


\section{Introduction}

Simulation procedures for fractional-order systems have been investigated in many current research projects. Such simulation procedures involve the numerically efficient and accurate evaluation of functions of the Mittag-Leffler type, the implementation of numerically efficient and robust simulation routines based on the Grünwald-Letnikov differentiation operator for linear and nonlinear system models, Laplace domain representations for the linear case, the development of frequency domain-based procedures and the design of software packages such as CRONE TOOLBOx. This toolbox includes computational routines for fractional-order time- and frequency-domain system identification, fractional-order path planning techniques, and approaches for a fractional-order control synthesis [10, 12, 13, 23, 27, 30].

Among the approaches mentioned above, the Grünwald-Letnikov operator for numerically approximating fractional-order derivatives and the corresponding numerical integration of fractional system models is widely used in engineering applications. It is based on a temporal series expansion of the fractional derivative operator and coincides, when setting the fractional differentiation order to one (i.e., considering classical first-order ordinary differential equations) to the well-known temporal Taylor series expansion of the solution to an ordinary differential equation that is typically truncated after some finite order in any numerical simulation of dynamic systems. The general drawback of this numerical evaluation scheme is the necessity for a large number of summands in the series expansion to capture the long-term memory effects of fractional systems with sufficient accuracy. Although the Grünald-Letnikov operator is generally applicable to linear as well as nonlinear fractional-order models, the large number of required terms in the series expansion prevents its naive use to general system models with uncertain parameters and initial conditions due to the inevitably arising wrapping effect. To avoid this wrapping effect, that also occurs if high-order series expansions are applied in the case of classical ordinary differential equations with purely integer-order derivatives, this paper is focused on using quasi-analytic representations of the enclosure of the systems' time responses by means of Mittag-Leffler functions [10]. Those functions represent a generalization of classical exponential functions and can be exploited - as shown for the first time in $[33,35]$ — to represent enclosures for the sets of reachable states for fractional-order systems if they are extended to the case of interval arguments.

Besides series expansions in the time domain, also frequency-domain approximations can be determined $[4,9,27,28,30]$. They make use of approximating the amplitude and phase responses by multiplicative concatenations (i.e., series concatenations in the respective signal flow) of fundamental linear Bode plot elements corresponding to first- and second-order lead and lag elements and, under some circumstances, input-output transport delays. As such, these frequency-domain techniques can be seen as the approximation of the fractional-order Laplace-domain transfer functions by using Taylor, respectively, Laurent series to approximate their numerators and denominators by expressions with integer-order powers of the Laplace variable (except for an isolated classical transport delay operator). Due 
to this strong relation to the Taylor and Laurent series expansion in the Laplace domain, the resulting integer-order approximations are only applicable to systems with dominating linear dynamics within a restricted frequency band. Although such approximations are commonly not suitable for accurate system simulations, they are well suited, if control design with sufficiently strong low-pass filter behavior is concerned and, for example, for the experimental identification of fractional models in restricted frequency bands with applications in engineering and biomedical tasks such as impedance spectroscopy for battery systems [2,38,39], rheological material properties [29], or the study of visco-elastic properties of blood cells [5].

Linear control approaches that are described by fractional-order transfer functions can be interpreted as extensions of classical output feedback routines of PID type (proportional, integrating, differentiating) by replacing the integrating and differentiating elements with their respective fractional-order generalizations [23, 27].

As already mentioned in the discussion of the Grünwald-Letnikov operator, the amount of memory required to accurately represent the flow of a fractionalorder system model may become prohibitively large if long integration horizons are considered. Hence, techniques for a short-term memory storage, going along with quantifying the errors arising from restarting the numerical integration of a fractional-order system at some specific point in time, are crucial in practice. In this paper, techniques for quantifying the effect of resetting the temporal derivative at some point of time will be used to describe guaranteed interval enclosures of the arising errors. These interval enclosures are then interfaced in a novel manner with the basic iteration approaches published in $[33,35]$ by combining them with the solution representations in terms of Mittag-Leffler functions. In such a way, the iterative solution scheme developed by the authors can be employed more efficiently for simulation scenarios in which long prediction horizons are of practical interest.

Although the numerical integration routines based on temporal series expansions, such as the Grünwald-Letnikov operator, are practically useful for a large variety of fractional-order system models that are characterized by point-valued system parameters and precisely known initial conditions, research concerning the analysis of uncertain but bounded parameters is still at the very beginning if fractional models are concerned. This problem has not yet received the same amount of attention as for the case of integer-order sets of ordinary differential equations. To the knowledge of the authors, only initial works were performed in this direction which are based on generalizations of the Picard iteration to the fractional case [22]. Using this iteration, it becomes possible to compute guaranteed outer interval enclosures for those states that are reachable over a sufficiently short prediction horizon. However, these enclosures - resulting from the integral formulation of the Picard iteration, see Theorem 3 in this paper - are typically quite conservative due to the fact that the resulting bounding boxes describe time-invariant state bounds that are valid for the complete prediction window.

In contrast to fractional-order systems, the task of verified simulation and reachability analysis has been studied extensively over the last decades in the frame of integer-order dynamic system models and corresponding control laws. Such techniques are readily applicable in terms of software-based simulation packages and 
can be employed - among others - for the verification of safety constraints of dynamic systems. State-of-the-art general-purpose initial value problem solvers for such tasks make use of so-called verified simulation approaches which are based on either interval analysis, zonotopic representations of the sets of reachable states, or Taylor model arithmetic $[26,33,36]$.

By means of set-valued computations, these solvers avoid time-consuming gridding techniques and Monte-Carlo sampling, where it has to be pointed out additionally that neither of these gridding and sampling techniques can provide any guarantee of determining outer solution tubes that contain the exact sets of reachable states of a general dynamic system model with absolute certainty. In contrast to grid- or sampling-based approaches, the fundamental property of those tubes computed with the help of verified approaches is that a guaranteed outer hull of the solutions of the underlying uncertain system model is determined [26]. To account for specific system properties such as a-priori proven asymptotic stability of the system dynamics (which is often verified in advance if a guaranteed stabilizing control design has been performed prior to evaluating the state equations), an exponential enclosure technique was developed by the working group of the authors for the class of integer-order systems [36]. Relations of this exponential enclosure approach to specific system properties such as cooperativity and positivity of a dynamic system [37] were published in [33]. If these latter properties are guaranteed to be satisfied, it becomes possible to evaluate lower and upper bounding trajectories independently during the numerical simulation. Such properties are often exploited during the design of interval observers which can analogously be derived for both, integer-order and fractional-order system representations [3, 8, 20,31].

If state equations are not a-priori proven to be cooperative, especially the use of the exponential enclosure technique allows for reducing overestimation (i.e., to avoid unphysically wide bounds for the computed state trajectories) in the case of asymptotically stable dynamics. This is caused by the fact that the exponential enclosure technique [33,35] aims at preserving stability properties in combination with a reduction of the wrapping effect [16]. As shown in previous work, this approach is most successful if a transformation of the state equations into a quasilinear system representation exists [35]. This transformation then has to ensure that the simulation routine makes use of a set of quasi-linear state equations given by a diagonally dominant form.

In this paper, the exponential enclosure technique is further developed for fractional-order systems, where exponential functions describing the guaranteed state enclosures have to be replaced by functions of the Mittag-Leffler type. The use of Mittag-Leffler functions as the corresponding ansatz for the solution enclosures is motivated by the fact that these functions represent the exact solutions of linear fractional-order models with precisely known parameters, cf. [7,11]. To allow for an efficient implementation of numeric simulation routines, preconditioning strategies of the state equations into a diagonally dominant form, the influence of truncation errors occurring from a finite-time approximation of the fractional-order systems' memory, and monotonicity properties of Mittag-Leffler functions with respect to its argument and with respect to the non-integer differentiation order need to be 
investigated. These aspects are discussed in detail in the current paper together with novel extensions towards the quantification of truncation errors resulting from infinite memory effects of fractional-order dynamics.

Following the summary of preliminaries and the state-of-the-art in Secs. 2 and 3, the exponential enclosure technique for interval-valued uncertain systems, which was so far primarily studied for classical sets of ordinary differential equations, is generalized to fractional-order models in Sec. 4. Here, we rely on the iteration procedure stated already in $[33,35]$. In the current paper, relations of this approach to the state-of-the-art, especially the integral formulation of the Picard iteration in Theorem 3, and extensions by a more detailed analysis of monotonicity properties allowing for an efficient implementation in an interval arithmetic framework together with handling temporal truncation errors are worked out as the novel contributions in Sec. 5. Sec. 6 provides illustrating linear and nonlinear examples for the use of the proposed enclosure technique before the paper is concluded with an outlook on future work in Sec. 7.

\section{Preliminaries}

In the course of this paper, simulation routines for the case of integer-order differential equations are first summarized. These routines are based on an intervalbased exponential enclosure technique. According to the corresponding publications in $[33,36]$, they result from a differential formulation of the Picard iteration. Second, they are generalized toward the counterpart of fractional-order dynamics. This generalization is essentially based on the replacement of exponential functions by suitable Mittag-Leffler functions as already motivated in [33, 35].

For that purpose, the two-parameter Mittag-Leffler function ${ }^{1}[10,12,14]$ is denoted by

$$
E_{\nu, \beta}(\zeta)=\sum_{i=0}^{\infty} \frac{\zeta^{i}}{\Gamma(\nu i+\beta)}
$$

with the general argument $\zeta \in \mathbb{C}$, the gamma function $\Gamma(\nu i+\beta)$, as well as the parameters $\nu \in \mathbb{R}_{+}$and $\beta \in \mathbb{R}$.

All system models in this paper are assumed to be given in terms of explicit, autonomous, time-invariant ${ }^{2}$ state equations which are re-written - if possible according to Def. 1 into a quasi-linear form to enhance efficiency of the numerical evaluation.

Definition 1 (Quasi-linear system model). After factoring out the state vector $\mathbf{x}(t) \in \mathbb{R}^{n}$ of a nonlinear autonomous system, initial value problems for quasi-linear

\footnotetext{
${ }^{1}$ The two-parameter Mittag-Leffler function serves as an exact solution representation for linear fractional-order differential equations according to $[7,11]$.

${ }^{2}$ Note, the restriction to autonomous, time-invariant systems can be removed by the introduction of auxiliary state variables for the time argument as well as for time- and state-dependent expressions included in control inputs. Corresponding procedures, leading to an increase of the system dimension, were discussed exemplarily in [36] for the integer-order case.
} 
models

$$
\dot{\mathrm{x}}(t)=\mathcal{A}(\mathbf{x}(t)) \cdot \mathbf{x}(t), \quad \mathcal{A}(\mathbf{x}(t)) \in \mathbb{R}^{n \times n},
$$

are specified with the vector of initial conditions

$$
\mathbf{x}(0) \in[\mathbf{x}](0)
$$

Analogously, a commensurate-order set of fractional-order differential equations of Caputo type [27,30] is defined by

$$
\mathbf{x}^{(\nu)}(t)=\mathcal{A}(\mathbf{x}(t)) \cdot \mathbf{x}(t) \text { with } 0<\nu<1
$$

where initial conditions $\mathbf{x}(0)$ are defined according to (3).

For both system models in Def. 1 , the initial state vector $\mathbf{x}(0)$ is assumed to be described by the interval representation $[\mathbf{x}](0)=[\underline{\mathbf{x}}(0) ; \overline{\mathbf{x}}(0)]$, where the inequalities $\underline{x}_{i}(0) \leq \bar{x}_{i}(0)$ hold element-wise for each vector component $i \in\{1, \ldots, n\}$.

The existence of a solution to the problem specified according to (4) with the initial conditions (3) is ensured if either of the iteration procedures in Secs. 3 or 4 converges to an appropriate interval enclosure.

Definition 2 (Diagonally dominant model). Diagonally dominant quasi-linear system models are given by the state-space representations

$$
\begin{aligned}
\dot{\mathbf{z}}(t)=\mathbf{f}(\mathbf{z}(t)) & =\left(\mathbf{T}^{-1} \cdot \mathcal{A}(\mathbf{T} \cdot \mathbf{z}(t)) \cdot \mathbf{T}\right) \cdot \mathbf{z}(t) \\
& =\mathbf{A}(\mathbf{z}(t)) \cdot \mathbf{z}(t), \quad \mathbf{A}(\mathbf{z}(t)) \in \mathbb{R}^{n \times n},
\end{aligned}
$$

and

$$
\mathbf{z}^{(\nu)}(t)=\mathbf{f}(\mathbf{z}(t))=\mathbf{A}(\mathbf{z}(t)) \cdot \mathbf{z}(t)
$$

after a suitable similarity transformation

$$
\mathbf{x}(t)=\mathbf{T} \cdot \mathbf{z}(t), \quad \mathbf{T} \in \mathbb{R}^{n \times n}, \quad \mathbf{z}(t) \in \mathbb{R}^{n}
$$

of the systems in Def. 1.

Remark 1. In this paper, we restrict ourselves to the case of real-valued similarity transformations in (7). These transformations lead to the real-valued initial state enclosures

$$
\mathbf{z}(0) \in \mathbf{T}^{-1} \cdot[\mathbf{x}](0)
$$

for both integer-order and fractional-order system models. As shown in [33,36] for integer-order system models, also complex-valued similarity transformations are possible. They are advantageous for the case of systems with conjugate-complex eigenvalues and, hence, oscillatory dynamics. For both the real- and complex-valued case with system models having an eigenvalue multiplicity of one, the transformation matrix $\mathbf{T}$ is composed of the eigenvectors of $\mathcal{A}\left(\mathbf{x}_{\mathrm{m}}\right)$, computed at the interval midpoint $\mathbf{x}_{\mathrm{m}}=\frac{1}{2} \cdot(\underline{\mathbf{x}}(0)+\overline{\mathbf{x}}(0))$. For generalizations to higher multiplicities, which were so far only investigated for integer-order scenarios, see [36]. 
Remark 2. Where necessary for a compact notation of the iteration formulas derived in the following sections, it is further assumed that a translation of the state vector has been performed prior to solving the considered simulation task so that the trajectories of the systems under consideration converge to the origin of the state space if the dynamics are asymptotically stable.

Example 1. Fractional-order differential equations appear, as stated in the introduction of this paper, in a variety of engineering applications. For example, series connections of electric subcircuits containing resistors and capacitors can be used for modeling the dynamics of Lithium-Ion batteries. The corresponding impedance (as the quotient between terminal voltage and current) then takes the form of the integer-order (IO) frequency response

$$
Z_{\mathrm{IO}}(\jmath \omega)=\frac{\sum_{i=0}^{n} b_{i} \cdot(\jmath \omega)^{i}}{\sum_{i=0}^{n} a_{i} \cdot(\jmath \omega)^{i}}
$$

with the imaginary unit $\jmath$ and the angular frequency $\omega \geq 0$. However, experimental impedance spectroscopy data gives rise to the more general fractional-order (FO) expression, see $[2,38,39]$,

$$
Z_{\mathrm{FO}}(\jmath \omega)=\frac{\sum_{i=0}^{n} b_{i} \cdot(\jmath \omega)^{\nu_{i}}}{\sum_{j=0}^{n} a_{j} \cdot(\jmath \omega)^{\nu_{j}}},
$$

where $\nu_{i}$ and $\nu_{j}$ are non-negative, not necessarily integer-valued parameters with $0 \leq \nu_{0}<\nu_{1}<\nu_{2}<\ldots$

Here, numerator expressions of order $\nu_{i}$ are related to fractional derivatives of the terminal current, while the orders $\nu_{j}$ in the denominator are connected with a non-integer derivative of the terminal voltage. Due to the fact that a repeated fractional-order differentiation, first of order $\nu_{a}$ and second of order order $\nu_{b}$ corresponds in total with a derivative of order $\nu_{a}+\nu_{b}$, see $[27,30]$, the type of system model mentioned in this example, can always be transferred into a commensurateorder state-space representation according to Defs. 1 and 2 by setting $\nu$ to the greatest common divisor of all fractional orders $\nu_{i}$ and $\nu_{j}$.

\section{State-of-the-Art Techniques Applicable to the Verified Simulation of Fractional-Order System Models}

\subsection{Exploitation of Differential Inclusions and Cooperativity}

Theorem 1 (Differential inclusions for fractional-order differential equations). Time-varying bounds for a fractional-order system described according to Def. 2 
are given by the interval vector

$$
\mathbf{z}(t) \in[\mathbf{v}(t) ; \mathbf{w}(t)]
$$

in which the individual components of the vectors $\mathbf{v}(t)$ and $\mathbf{w}(t)$ are solutions to the coupled lower and upper bounding systems

$$
\mathbf{v}^{(\nu)}(t)=\mathbf{f}_{v}(\mathbf{v}(t), \mathbf{w}(t)) \leq \mathbf{z}^{(\nu)}(t)=\mathbf{f}(\mathbf{z}(t)) \leq \mathbf{w}^{(\nu)}(t)=\mathbf{f}_{w}(\mathbf{v}(t), \mathbf{w}(t))
$$

representing differential inclusions for the dynamic system $\mathbf{z}^{(\nu)}(t)=\mathbf{f}(\mathbf{z}(t))$.

Proof. Theorem 1 is a straightforward consequence of Müller's theorem originally published for integer-order ordinary differential equations [25]. Substituting the integer-order derivatives in this theorem by their respective fractional-order counterparts completes the proof.

Corollary 1 (Differential inclusions for cooperative fractional-order differential equations). Time-varying bounds for cooperative, positive fractional-order systems described according to Def. 2 are given by the interval vector

$$
\mathbf{z}(t) \in[\mathbf{v}(t) ; \mathbf{w}(t)], \quad v_{i}(t) \geq 0, \quad i \in\{1, \ldots, n\},
$$

in which the individual components of the vectors $\mathbf{v}(t)$ and $\mathbf{w}(t)$ are solutions to the mutually decoupled lower and upper bounding systems

$$
\mathbf{v}^{(\nu)}(t)=\mathbf{f}(\mathbf{v}(t)) \leq \mathbf{z}^{(\nu)}(t)=\mathbf{f}(\mathbf{z}(t)) \leq \mathbf{w}^{(\nu)}(t)=\mathbf{f}(\mathbf{w}(t))
$$

Proof. Assume a cooperative dynamic system with strictly non-negative states $z_{i}(t) \geq 0$ satisfying the sufficient criterion for cooperativity $[8,17,32,37]$ given by

$$
J_{i, j}(\mathbf{z}) \geq 0 \quad \text { for all } \quad i \neq j, \quad i, j \in\{1, \ldots, n\} \quad \text { with } \quad \mathbf{J}=\frac{\partial \mathbf{f}(\mathbf{z})}{\partial \mathbf{z}} .
$$

An element-wise minimization (respectively, maximization) of the function $\mathbf{f}(\mathbf{z}(t)$ ) over the state interval (13) directly leads to its element-wise defined lower bound $\mathbf{f}(\mathbf{v}(t))$ (respectively, upper bound $\mathbf{f}(\mathbf{w}(t))$ ).

The property exploited in Corollary 1 is widely employed in the frame of observer design for both, integer-order and fractional-order system models. Suitable references concerning observer design as well as for its dual task, namely, cooperativitypreserving control synthesis can be found in $[8,15,20,32,34]$. If cooperativity is either directly given after first-principle modeling of the systems in Def. 1 or Def. 2, Corollary 1 provides overestimation-free state bounds if the element-wise minimizations and maximizations mentioned in the proof above coincide with actually reachable operating conditions. It has to be noted that in this case it is not necessary (from a practical point of view) to apply interval-based simulation routines as long as temporal discretization errors in the differential equations for $\mathbf{v}(t)$ and $\mathbf{w}(t)$ are 
negligibly small. For several practically relevant system models, such as the interval observer design for a fractional-order battery model in [15], cooperativity can be ensured by design. Alternatively, a cooperativity-enforcing change of variables can be performed to remove the restrictive assumptions imposed by cooperativity if a dynamic system model is initially not cooperative. Details about suitable transformation techniques are given in $[18,19,21]$. However, if cooperativity is either not given directly or cannot be achieved by these similarity transformations, the following alternatives need to be exploited to determine guaranteed bounds for all reachable states.

\subsection{Transformation of Fractional Systems into Equivalent Ordinary Differential Equations}

Theorem 2 ( [6] Solution of fractional-order differential equations by nonlinear time transformations). Let $\mathbf{f}(\mathbf{z}(t))$ be a bounded and continuous function. The solution to the time-invariant fractional-order differential equations considered in Def. 2 according to

$$
\mathbf{z}^{(\nu)}(t)=\mathbf{f}(\mathbf{z}(t))
$$

with the bounded initial conditions $\mathbf{z}(0)$ is given by

$$
\mathbf{z}(t)=\mathfrak{z}\left(\frac{t^{\nu}}{\Gamma(\nu+1)}\right)
$$

where $\mathfrak{z}(\tau)$ is determined as the solution to an initial value problem to the set of integer-order differential equations

$$
\frac{\mathrm{d} \mathfrak{z}(\tau)}{\mathrm{d} \tau}=\mathfrak{f}(\mathfrak{z}(\tau))
$$

with the initial condition

$$
\mathfrak{z}(0)=\mathbf{z}(0)
$$

and the nonlinear time transformation

$$
\tau=t-\left(t^{\nu}-\tau \cdot \Gamma(\nu+1)\right)^{\frac{1}{\nu}}
$$

leading to

$$
\mathfrak{f}(\mathfrak{z}(\tau))=\mathbf{f}\left(\mathbf{z}\left(t-\left(t^{\nu}-\tau \cdot \Gamma(\nu+1)\right)^{\frac{1}{\nu}}\right)\right),
$$

in which $\tau$ is the independent variable and $t$ is considered as a parameter.

Although Theorem 2 provides a quite general approach that makes initial value problem solvers originally developed for the case of integer-order differential equations applicable to the fractional-order case, it has two main drawbacks if uncertain systems are concerned: First, the nonlinear time transformation according to Theorem 2 leads to the fact that even for time-invariant system models, usually time-varying initial value problems need to be solved. In the general case, this 
can only be done by augmenting the state vector by the time variable $\tau$ according to the procedure discussed in [36], leading inevitably to an increase in the system dimension. Second, this augmentation of the state vector as well as the required backward transformation (17) of the computed solution usually introduce some additional amount of overestimation due to multiple dependencies on common interval variables.

Remark 3. The time-varying characteristics of the transformed system model in (21) with (20) highlights the property of fractional-order differential equations, that restarting the temporal solution procedure at some point of time $T>0$ purely on the basis of the novel initial conditions $\mathbf{z}(T)$ with simultaneously resetting the time to zero would inevitably lead to truncation errors. Handling of these errors by means of guaranteed error bounds on the derivative operator is discussed further in Secs. 5 and 6 of this paper.

\subsection{A Picard Iteration Procedure for Fractional-Order Dy- namics}

Theorem 3 ( $[1,22]$ Integral formulation of Picard iterations for fractional-order differential equations). Let $\mathbf{f}(\mathbf{z}(t))$ be a continuous Lipschitzian function on a bounded state and time domain. The solution to the time-invariant fractionalorder differential equations considered in Def. 2 at the point of time $T>0$ can be computed iteratively according to the fixed-point iteration

$$
\mathbf{z}^{\langle\kappa+1\rangle}(T):=\mathbf{z}(0)+\frac{1}{\Gamma(\nu)} \cdot \int_{0}^{T}(T-s)^{\nu-1} \cdot \mathbf{f}\left(\mathbf{z}^{\langle\kappa\rangle}(s)\right) \mathrm{d} s, \quad \kappa \in \mathbb{N}_{0},
$$

with the initialization $\mathbf{z}^{\langle 0\rangle}:=\mathbf{z}(0)$ at the iteration step $\kappa=0$.

This iteration generalizes to interval bounded initial conditions $\mathbf{z}(0) \in[\mathbf{z}](0)=$ $\left[\mathbf{z}_{0}\right]$ according to

$$
[\mathbf{z}]^{\langle\kappa+1\rangle}:=\left[\mathbf{z}_{0}\right]+\frac{1}{\Gamma(\nu+1)} \cdot\left[0 ; T^{\nu}\right] \cdot \mathbf{f}\left([\mathbf{z}]^{\langle\kappa\rangle}\right), \quad \kappa \in \mathbb{N}_{0},
$$

where convergence requires $[\mathbf{z}]^{\langle\kappa+1\rangle} \subseteq[\mathbf{z}]^{\langle\kappa\rangle}$, leading to $\mathbf{z}(t) \in[\mathbf{z}]^{\langle\kappa+1\rangle}$ for all $t \in$ $[0 ; T]$.

Theorem 3 provides the possibility to determine time-invariant bounds $[\mathbf{z}]^{\langle\kappa+1\rangle}$ containing all possible states $\mathbf{z}(t)$ that are reachable over the complete time interval $t \in[0 ; T]$. However, the fact that these bounds are time-invariant makes them excessively wide at the single point $t=T$. Hence, generalizations of this iteration are derived in the following section to obtain time-varying bounds which — for asymptotically stable dynamics — contract temporally towards the system's equilibrium state. 


\section{Interval-Based Iteration Procedure: Generalization of Exponential State Enclosures to Fractional-Order Systems}

In this section, an interval-based iteration procedure is derived for the computation of guaranteed state enclosures for fractional-order system models. To make this paper self-contained, an already existing variant for integer-order models as well as the initial work $[33,35]$ for the fractional-order case are briefly reviewed, before a detailed discussion about specific extensions to the fractional-order case is provided.

\subsection{Exponential State Enclosures for Integer-Order Ordi- nary Differential Equations}

Definition 3 (Exponential state enclosure). The time-dependent exponential enclosure function

$$
\mathbf{z}^{*}(t) \in\left[\mathbf{z}_{e}\right](t):=\exp ([\boldsymbol{\Lambda}] \cdot t) \cdot\left[\mathbf{z}_{e}\right](0), \quad\left[\mathbf{z}_{e}\right](0)=\left[\mathbf{z}_{0}\right]
$$

with the parameter matrix

$$
[\boldsymbol{\Lambda}]:=\operatorname{diag}\left\{\left[\lambda_{i}\right]\right\}, i \in\{1, \ldots, n\},
$$

is denoted as a verified exponential state enclosure for the system model (5) with (8) if it is determined according to Theorem 4.

Theorem 4 ( [36] Iteration for exponential state enclosures). The exponential state enclosure (24) is guaranteed to contain the set of all reachable states $\mathbf{z}^{*}(T)$ at the point of time $t=T>0$ according to

$$
\mathbf{z}^{*}(T) \in\left[\mathbf{z}_{e}\right](T):=\exp ([\boldsymbol{\Lambda}] \cdot T) \cdot\left[\mathbf{z}_{e}\right](0),
$$

if $[\boldsymbol{\Lambda}]$ is set to the outcome of the converging iteration

$$
\left[\lambda_{i}\right]^{\langle\kappa+1\rangle}:=\frac{f_{i}\left(\exp \left([\boldsymbol{\Lambda}]^{\langle\kappa\rangle} \cdot[t]\right) \cdot\left[\mathbf{z}_{e}\right](0)\right)}{\exp \left(\left[\lambda_{i}\right]^{\langle\kappa\rangle} \cdot[t]\right) \cdot\left[z_{e, i}\right](0)},
$$

$i \in\{1, \ldots, n\}$, with the prediction horizon $[t]=[0 ; T]$.

Proof. Assume that the integral form of the Picard iteration, see [36] and Theorem 3 with $\nu=1$,

$$
\mathbf{z}^{*}(t) \in\left[\mathbf{z}_{e}\right]^{\langle\kappa+1\rangle}:=\left[\mathbf{z}_{e}\right](0)+\int_{0}^{t} \mathbf{f}\left(\left[\mathbf{z}_{e}\right]^{\langle\kappa\rangle}(s)\right) \mathrm{d} s
$$

describes a converging iteration that encloses the exact solution $\mathbf{z}^{*}(t)$ to the initial value problem of an integer-order system as given in Def. 2 in terms of an outer 
interval hull over all possible state trajectories over the time horizon $t \in[t]=[0 ; T]$ with $T>0$.

The evaluation of the iteration (28) for the ansatz of an exponential state enclosure (24) with (25) according to Def. 3 yields the relation

$$
\begin{aligned}
\mathbf{z}^{*}(t) & \in \exp \left([\boldsymbol{\Lambda}]^{\langle\kappa+1\rangle} \cdot t\right) \cdot\left[\mathbf{z}_{e}\right](0) \\
& =\left[\mathbf{z}_{e}\right](0)+\int_{0}^{t} \mathbf{f}\left(\exp \left([\boldsymbol{\Lambda}]^{\langle\kappa\rangle} \cdot s\right) \cdot\left[\mathbf{z}_{e}\right](0)\right) \mathrm{d} s
\end{aligned}
$$

between the interval matrices $[\boldsymbol{\Lambda}]^{\langle\kappa\rangle}$ and $[\boldsymbol{\Lambda}]^{\langle\kappa+1\rangle}$ for the two subsequent iteration steps $\kappa$ and $\kappa+1$. The differentiation of $(29)$ with respect to time results in the differential form of the Picard iteration which is given by

$$
\begin{aligned}
\dot{\mathbf{z}}^{*}(t) & \in[\boldsymbol{\Lambda}]^{\langle\kappa+1\rangle} \cdot \exp \left([\boldsymbol{\Lambda}]^{\langle\kappa+1\rangle} \cdot t\right) \cdot\left[\mathbf{z}_{e}\right](0) \\
& =\mathbf{f}\left(\exp \left([\boldsymbol{\Lambda}]^{\langle\kappa\rangle} \cdot t\right) \cdot\left[\mathbf{z}_{e}\right](0)\right)=\mathbf{f}\left(\left[\mathbf{z}_{e}\right]^{\langle\kappa\rangle}(t)\right)
\end{aligned}
$$

with its corresponding interval extension for the complete prediction window $[t]$ according to

$$
\dot{\mathbf{z}}^{*}([t]) \in[\boldsymbol{\Lambda}]^{\langle\kappa+1\rangle} \cdot\left[\mathbf{z}_{e}\right]^{\langle\kappa+1\rangle}([t])=\mathbf{f}\left(\left[\mathbf{z}_{e}\right]^{\langle\kappa\rangle}([t])\right)
$$

All equivalent expressions (29)-(31) describe a converging iteration process if

$$
\left[\lambda_{i}\right]^{\langle\kappa+1\rangle} \subseteq\left[\lambda_{i}\right]^{\langle\kappa\rangle}
$$

and hence

$$
[\boldsymbol{\Lambda}]^{\langle\kappa+1\rangle} \subseteq[\boldsymbol{\Lambda}]^{\langle\kappa\rangle}
$$

are satisfied. Due to inclusion monotonicity [16] of the exponential function, the relations (32) and (33) imply

$$
\exp \left([\boldsymbol{\Lambda}]^{\langle\kappa+1\rangle} \cdot t\right) \subseteq \exp \left([\boldsymbol{\Lambda}]^{\langle\kappa\rangle} \cdot t\right)
$$

for all $t \in[t]$. Overapproximating the left-hand side of $(31)$, cf. [33,36], in a conservative manner with an interval

$$
\left[\lambda_{i}\right]^{\langle\kappa+1\rangle} \subseteq\left[\tilde{\lambda}_{i}\right]^{\langle\kappa+1\rangle} \subseteq\left[\lambda_{i}\right]^{\langle\kappa\rangle}
$$

according to

$$
\operatorname{diag}\left\{\left[\tilde{\lambda}_{i}\right]^{\langle\kappa+1\rangle}\right\} \cdot\left[\mathbf{z}_{e}\right]^{\langle\kappa\rangle}([t])=: \mathbf{f}\left(\left[\mathbf{z}_{e}\right]^{\langle\kappa\rangle}([t])\right)
$$

and solving the equality in (36) for the yet unknown bounds $\left[\tilde{\lambda}_{i}\right]^{\langle\kappa+1\rangle}$ with subsequently renaming this parameter into $\left[\lambda_{i}\right]^{\langle\kappa+1\rangle}$ completes the proof. 
For further discussions concerning the necessary zero-exclusion requirement $0 \notin\left[z_{e, i}\right]([t])$ for all components $i \in\{1, \ldots, n\}$ of the state vector as well as for generalizations to multiple real and/or complex eigenvalues, the reader is referred to [36]. Fundamental step-size control strategies and the definition of time-varying transformation matrices leading to less conservative quasi-linear system models than those in Def. 2 are given in [19].

Corollary 2. For quasi-linear state-space representations according to Def. 1, which are transformed into the diagonally dominant form of Def. 2, the componentwise notation

$$
\dot{z}_{i}(t)=f_{i}(\mathbf{z}(t))=\sum_{j=1}^{n} a_{i j}(\mathbf{z}(t)) \cdot z_{j}(t)
$$

of the state equations allows for a reduction of interval-related dependency problem, the wrapping effect, and the resulting computational effort if formula (27) is reformulated symbolically into

$$
\begin{aligned}
{\left[\lambda_{i}\right]^{\langle\kappa+1\rangle}:=} & a_{i i}\left(\left[\mathbf{z}_{e}\right]^{\langle\kappa\rangle}([t])\right) \\
& +\sum_{\substack{j=1 \\
j \neq i}}^{n}\left\{a_{i j}\left(\left[\mathbf{z}_{e}\right]^{\langle\kappa\rangle}([t])\right) \cdot e^{\left(\left(\left[\lambda_{j}\right]^{\langle\kappa\rangle}-\left[\lambda_{i}\right]^{\langle\kappa\rangle}\right) \cdot[t]\right)} \cdot \frac{\left[z_{e, j}\right](0)}{\left[z_{e, i}\right](0)}\right\} .
\end{aligned}
$$

\subsection{Mittag-Leffler Type State Enclosures for Fractional-Or- der Differential Equations}

The focus of this subsection is the generalization of the exponential enclosure technique to sets of commensurate fractional-order models. The fundamental iteration summarized in the following Theorem 5 was first published by the authors in [33] and [35]. The novelty of the present paper is the detailed description of relations to the state-of-the-art approaches in Sec. 3 and the in-depth discussion of intervalbased numerical evaluation schemes together with the reliable consideration of the infinite-horizon memory property that becomes crucial as soon as the integration time horizon is divided into several temporal subslices of finite duration.

Definition 4 (Mittag-Leffler type state enclosure). The time-dependent MittagLeffler type enclosure function

$$
\mathbf{z}^{*}(t) \in \mathbf{E}_{\nu, 1}\left([\boldsymbol{\Lambda}] \cdot t^{\nu}\right) \cdot\left[\mathbf{z}_{e}\right](0), \quad\left[\mathbf{z}_{e}\right](0)=\left[\mathbf{z}_{0}\right]
$$

with the diagonal parameter matrix $[\boldsymbol{\Lambda}]:=\operatorname{diag}\left\{\left[\lambda_{i}\right]\right\}, i \in\{1, \ldots, n\}$, is denoted as $a$ verified Mittag-Leffler type state enclosure for the system model (6) with (8) if it is determined according to Theorem 5.

Theorem 5 ( $[33,35]$ Iteration for Mittag-Leffler type enclosures). The MittagLeffler type state enclosure (39) is guaranteed to contain the set of all reachable states $\mathbf{z}^{*}(T)$ at the point of time $t=T>0$ according to

$$
\mathbf{z}^{*}(T) \in \mathbf{E}_{\nu, 1}\left([\boldsymbol{\Lambda}] \cdot T^{\nu}\right) \cdot\left[\mathbf{z}_{e}\right](0),
$$


if $[\boldsymbol{\Lambda}]$ is set to the outcome of the converging iteration

$$
\left[\lambda_{i}\right]^{\langle\kappa+1\rangle}:=\frac{f_{i}\left(\mathbf{E}_{\nu, 1}\left([\boldsymbol{\Lambda}]^{\langle\kappa\rangle} \cdot[t]^{\nu}\right) \cdot\left[\mathbf{z}_{e}\right](0)\right)}{E_{\nu, 1}\left(\left[\lambda_{i}\right]^{\langle\kappa\rangle} \cdot[t]^{\nu}\right) \cdot\left[z_{e, i}\right](0)},
$$

$i \in\{1, \ldots, n\}$, with the prediction horizon $[t]=[0 ; T]$.

Proof. According to $[7,11]$, the exact solution of a linear fractional-order differential equation

$$
z^{(\nu)}(t)=\lambda \cdot z(t)
$$

of Caputo type - for which only the initial conditions of the system states at $t=0$ are specified - is given by the analytic expression

$$
z(t)=E_{\nu, 1}\left(\lambda t^{\nu}\right) \cdot z(0) .
$$

As for the case of integer-order differential equations, this relation serves as an ansatz for describing verified state enclosures. By substituting it (cf. (30) in Theorem 5) into the differential formulation of the Picard iteration, which results from determining the fractional-order time derivative of the result in Theorem 3 , the expression

$$
\begin{aligned}
\mathbf{z}^{(\nu)}(t) & \in\left([\boldsymbol{\Lambda}]^{\langle\kappa+1\rangle}\right) \cdot \mathbf{E}_{\nu, 1}\left([\boldsymbol{\Lambda}]^{\langle\kappa+1\rangle} \cdot t^{\nu}\right) \cdot\left[\mathbf{z}_{e}\right](0) \\
& =\mathbf{f}\left(\mathbf{E}_{\nu, 1}\left([\boldsymbol{\Lambda}]^{\langle\kappa\rangle} \cdot t^{\nu}\right) \cdot\left[\mathbf{z}_{e}\right](0)\right)=: \mathbf{f}\left(\left[\mathbf{z}_{e}\right]^{\langle\kappa\rangle}([t])\right)
\end{aligned}
$$

is obtained.

Overapproximating the Mittag-Leffler type state enclosure $\mathbf{E}_{\nu, 1}\left([\boldsymbol{\Lambda}]^{\langle\kappa+1\rangle} \cdot t^{\nu}\right)$ in the iteration step $\kappa+1$ by the enclosure $\left[\mathbf{z}_{e}\right]^{\langle\kappa\rangle}([t])$ obtained in the previous iteration step on the left-hand side of (44), as it was also done in the proof of Theorem 4 for the integer-order counterpart, leads to

$$
\operatorname{diag}\left\{\left[\tilde{\lambda}_{i}\right]^{\langle\kappa+1\rangle}\right\} \cdot\left[\mathbf{z}_{e}\right]^{\langle\kappa\rangle}([t])=\mathbf{f}\left(\left[\mathbf{z}_{e}\right]^{\langle\kappa\rangle}([t])\right) .
$$

Solving this expression for $\left[\tilde{\lambda}_{i}\right]^{\langle\kappa+1\rangle}$ with subsequently renaming this parameter into $\left[\lambda_{i}\right]^{\langle\kappa+1\rangle}$ completes the proof of Theorem 5 .

Corollary 3. Quasi-linear state-space representations of fractional-order differential equations in diagonally dominant form according to Def. 2 can be simulated efficiently by the symbolically simplified iteration scheme

$$
\begin{aligned}
{\left[\lambda_{i}\right]^{\langle\kappa+1\rangle}:=} & a_{i i}\left(\left[\mathbf{z}_{e}\right]^{\langle\kappa\rangle}([t])\right) \\
& +\sum_{\substack{j=1 \\
j \neq i}}^{n}\left\{a_{i j}\left(\left[\mathbf{z}_{e}\right]^{\langle\kappa\rangle}([t])\right) \cdot \frac{E_{\nu, 1}\left(\left[\lambda_{j}\right]^{\langle\kappa\rangle} \cdot[t]^{\nu}\right)}{E_{\nu, 1}\left(\left[\lambda_{i}\right]^{\langle\kappa\rangle} \cdot[t]^{\nu}\right)} \cdot \frac{\left[z_{e, j}\right](0)}{\left[z_{e, i}\right](0)}\right\} .
\end{aligned}
$$


Remark 4. In contrast to Eq. (38) derived for integer-order systems, where analytic simplifications of exponential functions become possible, the quotient of two Mittag-Leffler functions in (46) cannot be simplified further in the general case. This imposes further restrictions on the numerical evaluation of (46) using techniques from interval arithmetic in the following section.

Remark 5. For the order $\nu=1$, the iteration formulas in Theorems 4 and 5 become identical due to $E_{1,1}(z) \equiv e^{z}$.

\section{$5 \quad$ Evaluating Mittag-Leffler Functions for Interval Arguments}

As described in [14], rough (outer) bounds for the evaluation of Mittag-Leffler functions with (real-valued) interval arguments can be determined by exploiting the property of a continuous interpolation between Gaussian (exponential) and Lorentzian (rational) functions according to

$$
\exp (-\zeta)<E_{\nu, 1}(-\zeta) \leq \frac{1}{1+\zeta}, \quad \zeta \geq 0
$$

in Fig. 1a and

$$
\exp \left(-\zeta^{2}\right)<E_{\nu, 1}\left(-\zeta^{2}\right) \leq \frac{1}{1+\zeta^{2}}, \quad \zeta \geq 0
$$

in Fig. 1b. However, if only subintervals from the range $\nu \in[0 ; 1]$ are required for a specific application scenario, these bounds are usually too conservative for the interval-based evaluation of the iteration formulas presented in the previous section.

Therefore, floating point evaluations of the Mittag-Leffler function using the MATLAB implementation of R. Garrappa [10] are extended in the following subsections to obtain tight guaranteed interval bounds for the case of real-valued arguments. The case of complex interval arguments is a topic for future research.

\subsection{Interval Evaluation of the Two-Parameter Mittag-Leffler Function with Real Arguments}

Theorem 6 ( [35] Interval bounds for the Mittag-Leffler function with real arguments). Interval evaluations of Mittag-Leffler functions with real-valued arguments $z \in[z]=[\underline{z} ; \bar{z}]$ are given by

$$
E_{\nu, \beta}([z]) \in\left[E_{\nu, \beta}^{*}([z])\right]=\left[\tilde{E}_{\nu, \beta}([z])\right]+\frac{\epsilon}{1+\epsilon} \cdot\left(1+\left|\left[\tilde{E}_{\nu, \beta}([z])\right]\right|\right) \cdot[-1 ; 1]
$$

with the tolerance value $\epsilon$ of a floating point function evaluation of (1) and the interval definition

$$
\left[\tilde{E}_{\nu, \beta}([z])\right]=\left[\nabla \tilde{E}_{\nu, \beta}(\underline{z}) ; \triangle \tilde{E}_{\nu, \beta}(\bar{z})\right]
$$




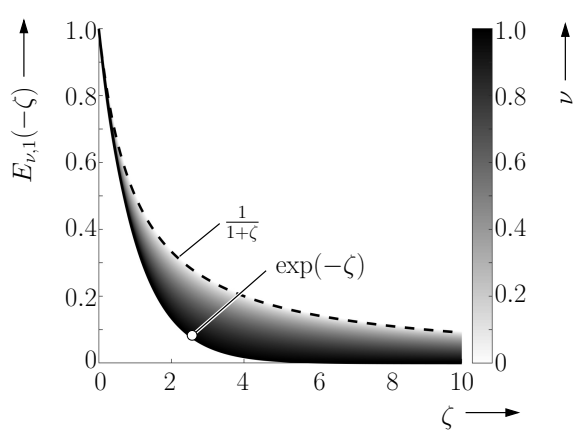

(a) Bounds for the Mittag-Leffer according to $(47)$.

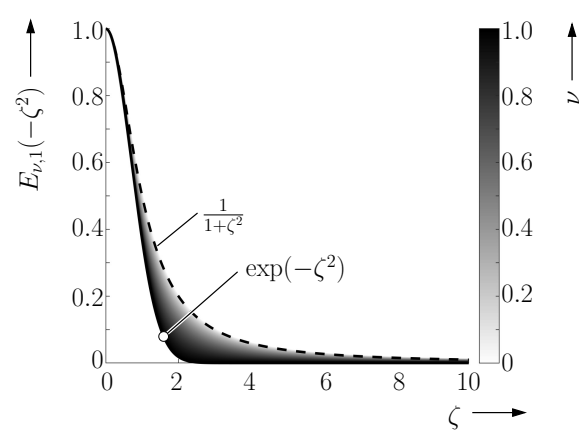

(b) Bounds for the Mittag-Leffer according to $(48)$.

Figure 1: Guaranteed bounds for Mittag-Leffler functions for the parameter range $\nu \in[0 ; 1]$, where the Lorentzian upper bound is highlighted by the dashed line and the gray color code visualizes the dependence on $\nu$.

where $\nabla$ and $\triangle$ denote switchings of the rounding mode of a CPU towards minus and plus infinity, respectively, in the corresponding floating point evaluations.

Proof. The interval extension (50) directly results form the strict monotonicity of the two-parameter Mittag-Leffler function with real-valued arguments. Moreover, a guaranteed tolerance value $\epsilon\left(\epsilon \approx 10^{-15}\right.$ for the case of the implementation by R. Garrappa [10]) allows to express the relative deviation between the floating point approximation $\tilde{E}_{\nu, \beta}(z)$ and the exact function value $E_{\nu, \beta}(z)$ at some value $z \in \mathbb{R}$, representable in floating point arithmetic, according to

$$
\frac{\left|E_{\nu, \beta}(z)-\tilde{E}_{\nu, \beta}(z)\right|}{1+\left|E_{\nu, \beta}(z)\right|}=\frac{|\Delta|}{1+\left|\tilde{E}_{\nu, \beta}(z)+\Delta\right|} \leq \epsilon .
$$

Solving the inequality (51) for $|\Delta|$ relies on the fact that

$$
\frac{|\Delta|}{1+\left|\tilde{E}_{\nu, \beta}(z)+\Delta\right|} \leq \frac{|\Delta|}{1+\left|\tilde{E}_{\nu, \beta}(z)\right|-|\Delta|}
$$

holds. Assuming

$$
\frac{|\Delta|}{1+\left|\tilde{E}_{\nu, \beta}(z)\right|-|\Delta|} \leq \epsilon
$$

in correspondence with (51) leads to the inequality

$$
|\Delta| \leq \frac{\epsilon}{1+\epsilon} \cdot\left(1+\left|\tilde{E}_{\nu, \beta}(z)\right|\right)
$$


which characterizes the interval bounds $[-1 ; 1] \cdot|\Delta|$ of the worst-case approximation error. Adding this tolerance interval to the outward rounded point-valued evaluation of the Mittag-Leffler function in Eq. (50) completes the proof of Theorem 6.

\subsection{Exploitation of Monotonicity in Interval Evaluations of the Mittag-Leffler Function}

To reduce overestimation in the interval evaluation of the iteration procedure according to Theorem 5, monotonicity properties of the Mittag-Leffer function with respect to the time $t$, the solution parameter $\lambda$ as well as to a derivative order $\nu$ specified as an interval variable are investigated in this section.

Theorem 7 ( [35] Monotonicity-based interval bounds for the Mittag-Leffler function). The range of function values for the Mittag-Leffler function with the uncertain real-valued parameters $\nu \in[\underline{\nu} ; \bar{\nu}], 0<\underline{\nu} \leq 1,0<\bar{\nu} \leq 1$ and $\lambda \in[\underline{\lambda} ; \bar{\lambda}]$, $\bar{\lambda}<0$ and the non-negative time argument $t \in[\underline{t} ; \bar{t}], \underline{t} \geq 0$, can be bounded tightly according to the interval enclosure

$$
E_{\nu, 1}\left(\lambda t^{\nu}\right) \in\left[E_{\bar{\nu}, 1}^{*}(\inf ([\mathcal{X}])) ; E_{\underline{\nu}, 1}^{*}(\sup ([\mathcal{X}]))\right]
$$

with $[\mathcal{X}]:=[\lambda] \cdot[t]^{[\nu]}$, where $\sup ([\mathcal{X}]) \leq 0$ holds.

If monotonicity with respect to $\nu$ can be proven additionally, the relation simplifies to

$$
E_{\nu, 1}\left(\lambda t^{\nu}\right) \in\left[E_{\bar{\nu}, 1}^{*}\left(\underline{\lambda} \cdot \bar{t}^{\bar{\nu}}\right) ; E_{\underline{\nu}, 1}^{*}\left(\bar{\lambda} \cdot \underline{t}^{\underline{\nu}}\right)\right]
$$

for the monotonically decreasing branch in $\nu$ and to

$$
E_{\nu, 1}\left(\lambda t^{\nu}\right) \in\left[E_{\underline{\nu}, 1}^{*}\left(\underline{\lambda} \cdot \bar{t}^{\underline{\nu}}\right) ; E_{\bar{\nu}, 1}^{*}\left(\bar{\lambda} \cdot \underline{t}^{\bar{\nu}}\right)\right]
$$

for the increasing branch; the change of monotonicity occurs on the surface depicted in Fig. 2.

Proof. Formula (55) is a direct consequence of the continuous interpolation property of Mittag-Leffler functions between Gaussian and Lorentzian functions, see the beginning of Sec. 5 and $[12,24]$. For a proof of monotonicity with respect to $t, \lambda$, and $\nu$, see [35].

Remark 6. For intervals $[\nu],[\lambda]$, and $[t]$ which do not intersect with the surface depicted in Fig. 2, the Eq. (56) holds for $\nu$ values below the surface (monotonically decreasing) and (57) for values above (monotonically increasing); otherwise (55) must be applied. 


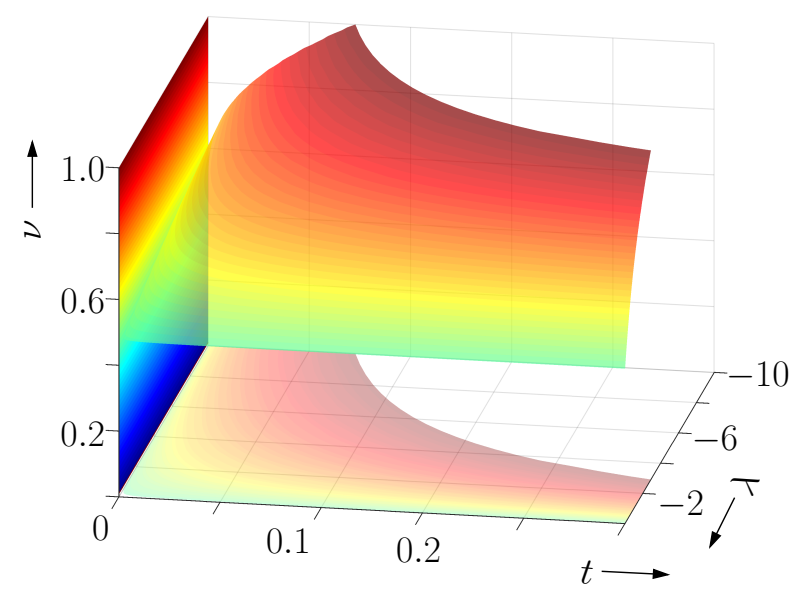

Figure 2: Surface, where the Mittag-Leffler function $E_{\nu, 1}\left(\lambda t^{\nu}\right)$ changes its monotonicity with respect to $\nu$.

\subsection{Interval Bounds for Temporal Truncation Errors due to the Infinite Memory Property of Fractional-Order Sys- tems}

It is well known that fractional-order system models are characterized by an infinite memory of previous states $[27,30]$. Hence, restarting a simulation at some point of time $t=t_{k+1}$ on the basis of state information $\mathbf{z}\left(t_{k+1}\right)$ computed by a simulation that was originally initiated at some point $t_{k}<t_{k+1}$ does not only have to account for these new initial conditions ${ }^{3}$. It also needs to consider the effect of temporal truncation errors which can be expressed by component-wise error bounds resulting from the fact that a fractional-order derivative of order $\nu$ with a memory start at $t=t_{k}$ is replaced with a new starting point $t=t_{k+1}$. The corresponding derivative operators are subsequently denoted by ${ }_{t_{k}} \mathcal{D}_{t}^{\nu} \mathbf{z}(t)$ and ${ }_{t_{k+1}} \mathcal{D}_{t}^{\nu} \mathbf{z}(t)$, respectively.

Theorem 8 (Bounds for temporal truncation errors). Resetting the initial point of time of the integration of fractional-order models defined in Def. 2 based on Theorem 5 after completion of a time interval of length $T$ requires the inflation of the right-hand side of the state equations by the symmetric interval $[-\boldsymbol{\mu} ; \boldsymbol{\mu}]$ at the point $T$ with

$$
\boldsymbol{\mu}:=\frac{\mathcal{Z} \cdot\left(t_{k}+T\right)^{-\nu}}{|\Gamma(1-\nu)|}
$$

\footnotetext{
${ }^{3}$ For the sake of compactness, the notation in this subsection is based on Def. 2. A transfer towards Def. 1 solely requires to replace all occurrences of the vectors $\mathbf{z}(t)$ with their counterpart $\mathbf{x}(t)$.
} 
and the component-wise defined supremum of the set of reachable states

$$
\mathcal{Z}_{i}=\sup _{t \in\left[t_{0} ; t_{k+1}\right]}\left|z_{i}(t)\right|
$$

The re-initialized initial value problem is then given by

$$
{ }_{t_{k}+T} \mathcal{D}_{t}^{\nu} \mathbf{z}(t)=\mathbf{z}^{(\nu)}(t)=\mathbf{f}(\mathbf{z}(t))+[-\boldsymbol{\mu} ; \boldsymbol{\mu}]=: \tilde{\mathbf{f}}(\mathbf{z}(t))
$$

with the initial state enclosure $\mathbf{z}\left(t_{k}+T\right) \in[\mathbf{z}]\left(t_{k}+T\right)$ resulting form the solution of

$$
{ }_{t_{0}} \mathcal{D}_{t}^{\nu} \mathbf{z}(t)=\mathbf{z}^{(\nu)}(t)=\mathbf{f}(\mathbf{z}(t)) \quad \text { with } \quad \mathbf{z}\left(t_{0}\right) \in[\mathbf{z}]\left(t_{0}\right), \quad t_{0}=0
$$

Proof. Theorem 8 is a consequence of the component-wise defined error bounds for a general fractional derivative operator of a commensurate system model on the time interval $t_{k}+T \leq t \leq t_{k+1}$ that can be computed according to [30] by

$$
\left|{ }_{t_{k}} \mathcal{D}_{t}^{\nu} \mathbf{z}(t)-{ }_{t_{k}+T} \mathcal{D}_{t}^{\nu} \mathbf{z}(t)\right| \leq \frac{\mathcal{Z} T^{-\nu}}{|\Gamma(1-\nu)|}=: \boldsymbol{\mu}
$$

As presented in [30], Eq. (62) relies on the component-wise defined supremum (59) of the reachable states denoted by the vector $\mathcal{Z}$.

A visualization of this state resetting procedure, with a corresponding adjustment of the right-hand side of the set of state equations is given in the following section. The following section accounts both for linear and nonlinear system models, as well as for a first possibility to interface the bounding approach according to Eq. (62) with a contractor technique [16] applied to the solution parameters $\left[\lambda_{i}\right]$ that for some system models yields tighter bounds than those given purely by applying the iteration of Theorem 5 after inflating the right-hand sides of the state equation with the vector $\boldsymbol{\mu}$. The reason for these possible enhancements can be seen in the fact that the original bound $\boldsymbol{\mu}$ captures an infinitely long time window starting at $t=t_{k}$, while in many practical scenarios much shorter windows are sufficient for the reliable forecast of the set of all possible state trajectories.

Theorem 9 (Contractor for the state enclosure of fractional-order systems). Assume that a reference solution $\mathbf{z}(t) \in\left[\mathbf{z}_{\mathrm{ref}}\right](t)$ has already been computed for the initial value problem with the initial point of time $t=t_{k}$ that is valid up to the point $t=t^{*}>t_{k}+T$ and that the application of Theorem 5 to the re-initialized initial value problem (60) in Theorem 8 with the initial point of time $t=t_{k}+T$ has provided the interval bounds $\left[\mathbf{z}_{e}\right](t)=\exp \left([\boldsymbol{\Lambda}] \cdot\left(t-\left(t_{k}+T\right)\right)\right) \cdot\left[\mathbf{z}_{e}\right]\left(t_{k}+T\right)$ that are also valid up to $t=t^{*}$ with the associated solution parameters $\left[\lambda_{i}\right], i \in\{1, \ldots, n\}$, a contractor is given by

$$
\left[\lambda_{i}\right]:=\left[\lambda_{i}\right] \cap\left[\tilde{\lambda}_{i}\right]
$$

with

$$
\left[\tilde{\lambda}_{i}\right]=\frac{\tilde{f}_{i}\left(\left[\mathbf{z}_{e}\right]\left(\left[t_{k}+T ; t^{*}\right]\right)\right) \cap \tilde{f}_{i}\left(\left[\mathbf{z}_{\mathrm{ref}}\right]\left(\left[t_{k}+T ; t^{*}\right]\right)\right)}{\left[z_{e, i}\right]\left(\left[t_{k}+T ; t^{*}\right]\right) \cap\left[z_{i, \mathrm{ref}}\right]\left(\left[t_{k}+T ; t^{*}\right]\right)} .
$$


Proof. The validity of Theorem 9 is a direct consequence of the fact that both $\left[\mathbf{z}_{\text {ref }}\right](t)$ and $\left[\mathbf{z}_{e}\right](t)$ are verified state enclosures according to $\mathbf{z}(t) \in\left[\mathbf{z}_{\text {ref }}\right](t)$ and $\mathbf{z}(t) \in\left[\mathbf{z}_{e}\right](t)$ and, thus, have to satisfy the fractional-order differential equation in the componentwise notation (44). Intersecting the evaluation of (44) for both state enclosures after consideration of the error bounds $\boldsymbol{\mu}$ for the corresponding point of restart and solving the result for the interval of the solution parameter $\left[\tilde{\lambda}_{i}\right]$ yields the relation (64).

\section{Illustrating Examples}

\subsection{Visualization of Interval Bounds for Temporal Trunca- tion Errors of Linear Fractional-Order Systems}

To visualize the influence of temporal truncation errors on the solution quality, consider the Caputo type linear fractional-order differential equation

$$
z^{(\nu)}(t)=-z(t)
$$

with the differentiation order $\nu=0.5$ as well as the initial state $z(0)=1$. According to (42) and (43) its exact solution is given by

$$
z(t)=E_{0.5,1}\left(-t^{0.5}\right) \quad \text { for } \quad t \geq 0 .
$$

This solution is visualized in Fig. 3 .

In addition, assume that the overall integration time horizon $t \in\left[0 ; T_{\mathrm{f}}\right]$ with $T_{\mathrm{f}}=10$ for this system is split into $N$ equidistant slices $\left[\tau_{k}\right]:=\left[t_{k-1} ; t_{k}\right]$ with $t_{0}=0$ and $t_{k}=k \cdot \frac{T_{\mathrm{f}}}{N}, k \in\{1, \ldots, N\}$. Neglecting the infinite-time horizon memory of this system, approximate solutions $\tilde{z}(t)$ are computed recursively by means of

$$
\tilde{z}(t)=\left(E_{0.5,1}\left(-\left(\frac{T_{\mathrm{f}}}{N}\right)^{0.5}\right)\right)^{k-1} \cdot E_{0.5,1}\left(-\left(t-t_{k-1}\right)^{0.5}\right)
$$

where $\tilde{z}(0)=z(0)$ and $t \in\left[\tau_{k}\right]$. As shown in Fig. 3, the quality of these approximations becomes worse, the larger the value $N$ is chosen.

To quantify the effect of the infinite-horizon memory, the error quantification according to Sec. 5.3 is included in the iteration scheme for determining the parameter $[\lambda]$ according to Theorem 5. It becomes obvious that the interval enclosures included in Fig. 4 for the time steps $k \geq 2$ contain the exact solution to the initial value problem. Note, the computation of the parameter enclosures $[\lambda]$ according to Theorem 5 was interfaced with intersecting the iteration result with a further conservative expression obtained by

$$
[\tilde{\lambda}]:=\frac{-\left[z_{e}\right]\left(\left[\tau_{k}\right]\right)+[-1 ; 1] \cdot \mu\left(t_{k-1}\right)}{\left(E_{0.5,1}\left(-\left[\tau_{k}\right]^{0.5}\right) \cdot[1-\eta ; 1+\eta]\right) \cap\left[z_{e}\right]\left(\left[\tau_{k}\right]\right)}, \quad 0<\eta<1
$$

as a special case of Theorem 9, where the numerator directly results from computing the state enclosure as shown in (41) and the denominator includes some a-priori knowledge on the domain of reachable states in the time interval $\left[\tau_{k}\right]$. 


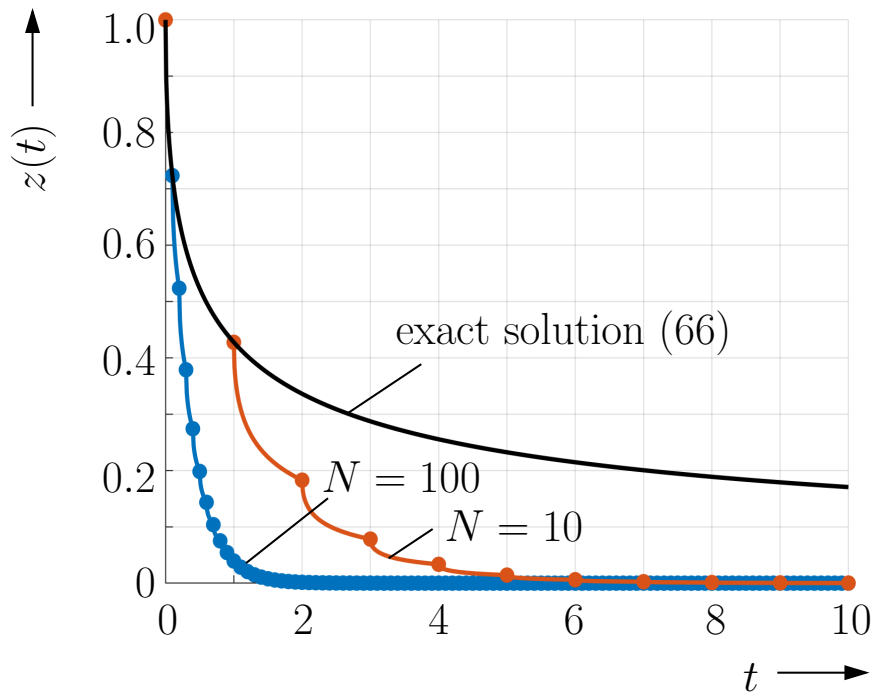

Figure 3: Visualization of truncation errors resulting from the infinite-horizon memory effect of fractional-order systems.

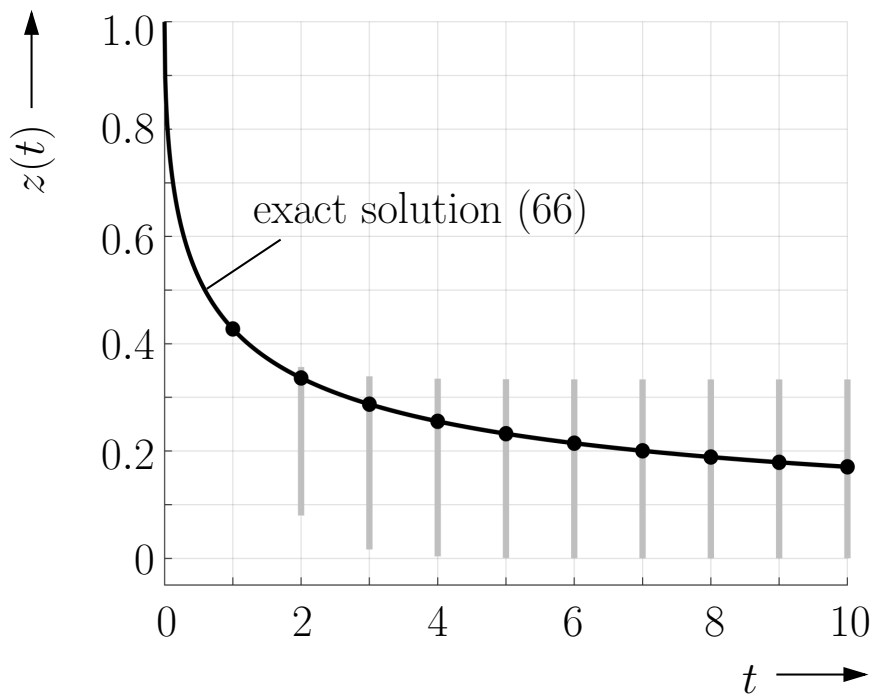

Figure 4: Interval-based quantification of truncation errors resulting from the infinite-horizon memory effect of fractional-order systems with $\eta=0.1$. 


\subsection{A Nonlinear Example: Interval Bounds for Different In- tegration Horizons}

As a second, nonlinear example for the application of Theorem 5 , the state equation

$$
z^{(\nu)}(t)=p \cdot z^{3}(t)=p \cdot a(z(t)) \cdot z(t)
$$

with an uncertain initial state $z(0) \in[z](0)$, the interval parameter $p \in[p]$, and the uncertain differentiation order $\nu \in[\nu]$ is considered. Note that Eq. (69) already includes the reformulation into a quasi-linear system model so that the modified iteration formula (46) becomes applicable.

In the following, two cases differing in the amount of uncertainty according to

Case a: $[z](0)=[0.99 ; 1.0],[p]=[-2 ;-1.99],[\nu]=[0.8 ; 0.81]$

and

Case b: $[z](0)=[0.5 ; 1.0],[p]=[-2 ;-1],[\nu]=[0.8 ; 0.9]$

are distinguished for this example.

Using the integration time horizons $T \in\{0.25,0.50,1.0\}$ for both Case a and Case b, without restarting the integration at any point in the interior of the temporal window $t \in[0 ; T]$, the state enclosures in Figs. 5a and 5b are obtained. It can be noticed that for both cases the iteration describes non-diverging state enclosures despite the fact that constant parameter bounds $[\lambda]$ were determined for the complete integration time horizons. Both, for small uncertainty levels in Fig. $5 \mathrm{a}$ and large uncertainty in Fig. 5b, these computed interval bounds become wider for increasing lengths of the integration horizon, due to the fact that solutions close to the steady state need to be incorporated. This statement can also be verified by investigating the numerical parameter values produced by Theorem 5 (listed in ascending order of $T$ ) for

Case a: $[\lambda] \in\{[-2.0001 ;-0.5261],[-2.0001 ;-0.2276],[-2.0001 ;-0.0684]\}$

as well as for

Case b: $[\lambda] \in\{[-2.0001 ;-0.0667],[-2.0001 ;-0.0270],[-2.0001 ;-0.0066]\}$.

Considering again the Case a, a restart of the integration is now performed at the points $t_{k} \in\{0.25,0.50,0.75\}$. The resulting state enclosure, including a comparison with the bounds obtained for a single time window of length $T=1$ are shown in Fig. 5c. Here, the contractor introduced in Theorem 9 has the form

$$
[\tilde{\lambda}]:=\frac{-p \cdot\left(\left[z_{e}\right]\left(\left[\tau_{k}\right]\right) \cap\left[z_{\mathrm{ref}}\right]\left(\left[\tau_{k}\right]\right)\right)^{3}+[-1 ; 1] \cdot \mu\left(t_{k-1}\right)}{\left[z_{e}\right]\left(\left[\tau_{k}\right]\right) \cap\left[z_{\mathrm{ref}}\right]\left(\left[\tau_{k}\right]\right)},
$$

where $\left[z_{\mathrm{ref}}\right]\left(\left[\tau_{k}\right]\right)$ is the evaluation of the enclosure function obtained for the overall time window without any temporal discretization. It can be seen that the subdivision of the time window $(T=1)$ leads to a noticeable reduction of the computed 


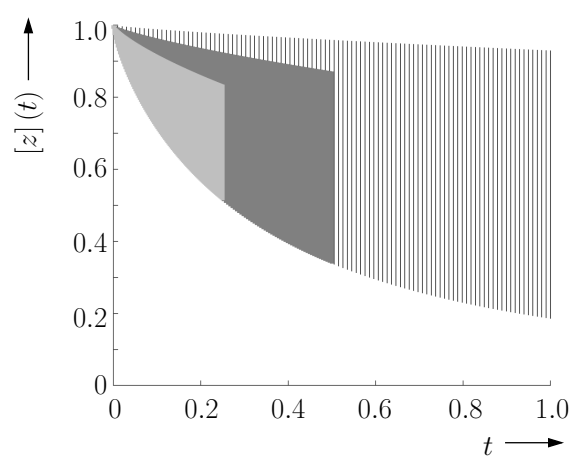

(a) State enclosures for Case a.

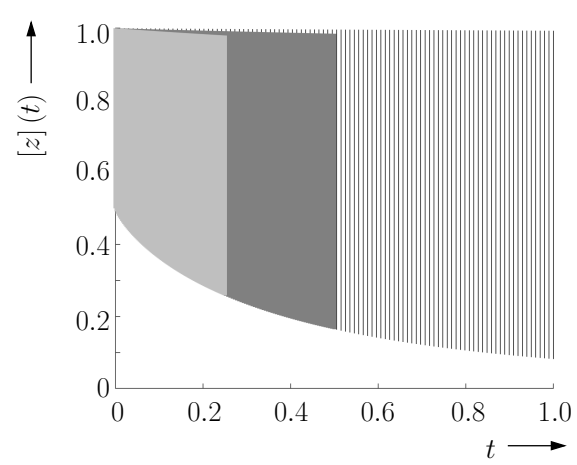

(b) State enclosures for Case b.

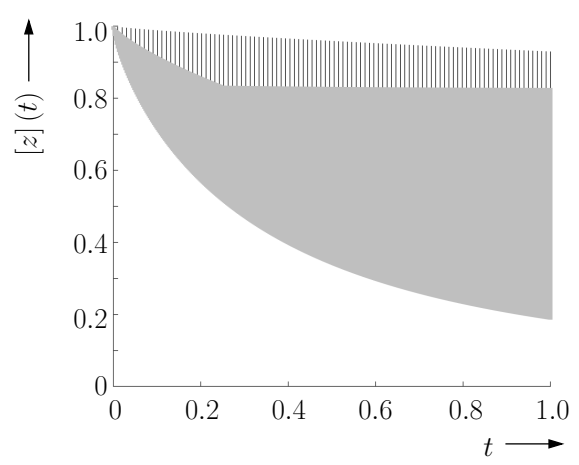

(c) State enclosures for Case a, including a restart of the integration at the points $t_{k} \in$ $\{0.25,0.50,0.75\}$.

Figure 5: Guaranteed state enclosures for different integration horizons $T \in$ $\{0.25,0.50,1.0\}$ and different levels of uncertainty.

interval widths. Future work will aim at the development of further contractor approaches, allowing both for a refinement of the bounds $\boldsymbol{\mu}$ and for incorporating simulations computed over long time windows as some kind of measured state enclosure as it would be done in the frame of an interval-based state observer synthesis. To perform a comparison with the parameter bounds listed above, the following results were obtained: $[\lambda]\left(\left[\tau_{1}\right]\right) \in[-2.0001 ;-0.5261],[\lambda]\left(\left[\tau_{2}\right]\right) \in[-3.3658 ;-0.0121]$, $[\lambda]\left(\left[\tau_{3}\right]\right) \in[-2.6236 ;-0.0064]$, and $[\lambda]\left(\left[\tau_{4}\right]\right) \in[-2.1951 ;-0.0035]$ with $\left[\tau_{1}\right]=$ $[0 ; 0.25],\left[\tau_{2}\right]=[0.25 ; 0.50],\left[\tau_{3}\right]=[0.50 ; 0.75]$, and $\left[\tau_{4}\right]=[0.75 ; 1.0]$. 


\section{Conclusions and Outlook on Future Work}

In this paper, extensions of an interval-based exponential enclosure technique originally developed for integer-order sets of ordinary differential equations were presented to obtain a novel Mittag-Leffler function-based generalization valid also for explicit, continuous-time sets of fractional-order differential equations. This type of iteration was first discussed by the authors in $[33,35]$, however, without accounting for the practically necessary extension towards the use of temporal subintervals. The corresponding time discretization scheme requires the quantification of truncation errors - which are caused by the infinite-horizon memory effects of fractional-order systems - and which do not exist for classical ordinary differential equations.

A first implementation of the novel routine for quantifying these error bounds, has been presented and interfaced for the first time with a contractor approach that further allows for reducing conservativeness of the obtained solution sets.

Future work will deal with a generalization of the iteration scheme to system models with an oscillatory behavior, for which it seems to be reasonable that complex-valued state enclosures are determined as it was already demonstrated for the case of the integer-order counterpart [36]. Moreover, possible strategies for determining optimal subdivision strategies of the investigated integration time horizons — with the aim of minimizing the computed interval diameters — will be investigated.

\section{References}

[1] Amairi, M., Aoun, M., Najar, S., and Abdelkrim, M.N. A constant enclosure method for validating existence and uniqueness of the solution of an initial value problem for a fractional differential equation. Applied Mathematics and Computation, 217(5):2162-2168, 2010. DOI: 10.1016/j . amc.2010.07.015.

[2] Andre, D., Meiler, M., Steiner, K., Wimmer, Ch., Soczka-Guth, T., and Sauer, D.U. Characterization of High-Power Lithium-Ion Batteries by Electrochemical Impedance Spectroscopy. I. Experimental Investigation. Journal of Power Sources, 196(12):5334-5341, 2011. DOI: 10.1016/j.jpowsour .2010.12.102.

[3] Bel Haj Frej, G., Malti, R., Aoun, M., and Raïssi, T. Fractional Interval Observers And Initialization Of Fractional Systems. Communications in Nonlinear Science and Numerical Simulation, 82:105030, 2020. DOI: 10.1016/j.cnsns.2019.105030.

[4] Chen, Y. Oustaloup-Recursive-Approximation for Fractional Order Differentiators. MATLAB Central File Exchange. www. mathworks.com/matlabcent $\mathrm{ral} /$ fileexchange/3802-oustaloup-recursive-approximation-for-fra ctional-order-differentiators, accessed: Aug. 14, 2020. 
[5] Craiem, D. and Magin, R. Fractional Order Models of Viscoelasticity as an Alternative in the Analysis of Red Blood Cell (RBC) Membrane Mechanics. Physical Biology, 7:13001, 03 2010. DOI: 10.1088/1478-3975/7/1/013001.

[6] Demirci, E. and Ozalp, N. A method for solving differential equations of fractional order. Journal of Computational and Applied Mathematics, 236(11):2754-2762, 2012. DOI: 10.1016/j.cam.2012.01.005.

[7] Dorjgotov, K., Ochiai, H., and Zunderiya, U. On Solutions of Linear Fractional Differential Equations and Systems Thereof. 2018. arXiv:1803.09063.

[8] Efimov, D., Raïssi, T., Chebotarev, S., and Zolghadri, A. Interval State Observer for Nonlinear Time Varying Systems. Automatica, 49(1):200-205, 2013. DOI: $10.1016 / j$.automatica.2012.07.004.

[9] El-Khazali, R., Batiha, I.M., and Momani, S. Approximation of fractionalorder operators. In Agarwal, P., Baleanu, D., Chen, Y., Momani, S., and Machado, J.A.T., editors, Fractional Calculus. ICFDA 2018. Springer Proceedings in Mathematics \& Statistics, vol. 303, pages 121-151, Singapore, 2019. Springer Singapore. DOI: 10.1007/978-981-15-0430-3_8.

[10] Garrappa, R. Numerical Evaluation of Two and Three Parameter MittagLeffler Functions. SIAM Journal on Numerical Analysis, 53(3):1350-1369, 2015. DOI: $10.1137 / 140971191$.

[11] Ghosh, U., Sarkar, S., and Das, S. Solution of System of Linear Fractional Differential Equations with Modified Derivative of Jumarie Type. American Journal of Mathematical Analysis, 3(3):72-84, 2015. DOI: 10.12691/ajma $-3-3-3$.

[12] Gorenflo, R., Kilbas, A.A., Mainardi, F., and Rogosin, S.V. Mittag-Leffler Functions, Related Topics and Applications. Springer-Verlag, Berlin, Heidelberg, 2014. DOI: 10.1007/978-3-662-43930-2.

[13] Gorenflo, R., Loutchko, J., and Luchko, Y. Computation of the Mittag-Leffler Function and its Derivatives. Fractional Calculus $\&$ Applied Analysis (FCAA), $5(4): 491-518,2002$.

[14] Haubold, H.J., Mathai, A.M., and Saxena, R.K. Mittag-Leffler Functions and Their Applications. Journal of Applied Mathematics, 2011:51 pages, 2011. DOI: $10.1155 / 2011 / 298628$.

[15] Hildebrandt, E., Kersten, J., Rauh, A., and Aschemann, H. Robust Interval Observer Design for Fractional-Order Models with Applications to State Estimation of Batteries. In Proc. of the 21st IFAC World Congress, Berlin, Germany, 2020.

[16] Jaulin, L., Kieffer, M., Didrit, O., and Walter, É. Applied Interval Analysis. Springer-Verlag, London, 2001. DOI: 10.1007/978-1-4471-0249-6. 
[17] Kaczorek, T. Positive 1D and 2D Systems. Springer-Verlag, London, 2002. DOI: $10.1007 / 978-1-4471-0221-2$.

[18] Kersten, J., Rauh, A., and Aschemann, H. State-Space Transformations of Uncertain Systems with Purely Real and Conjugate-Complex Eigenvalues into a Cooperative Form. In Proc. of 23rd Intl. Conference on Methods and Models in Automation and Robotics, Miedzyzdroje, Poland, 2018. DOI: 10.1109/MM AR. 2018.8486085.

[19] Kersten, J., Rauh, A., and Aschemann, H. Application-Based Discussion of Verified Simulations of Interval Enclosure Techniques. In Proc. of 24th Intl. Conference on Methods and Models in Automation and Robotics, Miedzyzdroje, Poland, 2019. DOI: 10.1109/MMAR.2019.8864673.

[20] Kersten, J., Rauh, A., and Aschemann, H. Transformation of Uncertain Linear Fractional Order Differential Equations into a Cooperative Form. In Proc. of the 8th IFAC Symposium on Mechatronic Systems (MECHATRONICS 2019) and the 11th IFAC Symposium on Nonlinear Control Systems (NOLCOS 2019), Vienna, Austria, 2019. DOI: 10.1016/j.ifacol.2019.12.035.

[21] Kersten, J., Rauh, A., and Aschemann, H. Application-Based Analysis of Transformations of Uncertain Dynamical Systems Into a Cooperative Form. Reliable Computing, 2020. Under review.

[22] Lyons, R., Vatsala, A.S., and Chiquet, R. Picard's Iterative Method for Caputo Fractional Differential Equations with Numerical Results. Mathematics, 5(4), 2017. DOI: $10.3390 /$ math5040065.

[23] Malti, R. and Victor, S. CRONE Toolbox for System Identification Using Fractional Differentiation Models. In Proc. of 17th IFAC Symposium on System Identification SYSID 2015, volume 48, pages 769-774, 2015. DOI: 10.1016/ j.ifacol.2015.12.223.

[24] Miller, K.S. and Samko, S.G. A Note on the Complete Monotonicity of the Generalized Mittag-Leffler Function. Real Analysis Exchange, 23(2):753-756, 1997-98. DOI: $10.2307 / 44153996$.

[25] Müller, M. Über die Eindeutigkeit der Integrale eines Systems gewöhnlicher Differenzialgleichungen und die Konvergenz einer Gattung von Verfahren zur Approximation dieser Integrale. In Sitzungsbericht Heidelberger Akademie der Wissenschaften, 1927. In German.

[26] Nedialkov, N.S. Interval Tools for ODEs and DAEs. In CD-Proc. of 12th GAMM-IMACS Intl. Symposium on Scientific Computing, Computer Arithmetic, and Validated Numerics SCAN 2006, Duisburg, Germany, 2007. IEEE Computer Society. DOI: 10.1109/SCAN.2006.28.

[27] Oustaloup, A. La Dérivation Non Entière: Théorie, Synthèse et Applications. Hermès, Paris, 1995. In French. 
[28] Oustaloup, A., Levron, F., Mathieu, B., and Nanot, F. M. Frequency-Band Complex Noninteger Differentiator: Characterization and Synthesis. IEEE Transactions on Circuits and Systems I: Fundamental Theory and Applications, 47(1):25-39, 2000. DOI: 10.1109/81.817385.

[29] Papoulia, K., Panoskaltsis, V., Kurup, N., and Korovajchuk, I. Rheological Representation of Fractional Order Viscoelastic Material Models. Rheologica Acta, 49:381-400, 04 2010. DOI: 10.1007/s00397-010-0436-y.

[30] Podlubny, I. Fractional Differential Equations: An Introduction to Fractional Derivatives, Fractional Differential Equations, to Methods of Their Solution and Some of Their Applications. Mathematics in Science and Engineering. Academic Press, London, 1999. DOI: 10.1016/s0076-5392(99)x8001-5.

[31] Raïssi, T. and Efimov, D. Some Recent Results on the Design and Implementation of Interval Observers for Uncertain Systems. at-Automatisierungstechnik, 66(3):213-224, 2018. DOI: 10.1515/auto-2017-0081.

[32] Raïssi, T., Efimov, D., and Zolghadri, A. Interval State Estimation for a Class of Nonlinear Systems. IEEE Transactions on Automatic Control, 57:260-265, 2012. DOI: 10.1109/TAC.2011.2164820.

[33] Rauh, A., Kersten, J., and Aschemann, H. Techniques for Verified Reachability Analysis of Quasi-Linear Continuous-Time Systems. In Proc. of 24th Intl. Conference on Methods and Models in Automation and Robotics, Miedzyzdroje, Poland, 2019. DOI: 10.1109/MMAR.2019.8864648.

[34] Rauh, A., Kersten, J., and Aschemann, H. Interval and Linear Matrix Inequality Techniques for Reliable Control of Linear Continuous-Time Cooperative Systems with Applications to Heat Transfer. International Journal of Control, pages 1-18, 2020. DOI: 10.1080/00207179.2019.1708966, Available online.

[35] Rauh, A., Kersten, J., and Aschemann, H. Interval-Based Verification Techniques for the Analysis of Uncertain Fractional-Order System Models. In Proc. of the 18th European Control Conference ECC2020, St. Petersburg, Russia, 2020.

[36] Rauh, A., Westphal, R., Aschemann, H., and Auer, E. Exponential Enclosure Techniques for Initial Value Problems with Multiple Conjugate Complex Eigenvalues. In Nehmeier, M., von Gudenberg, J. Wolff, and Tucker, W., editors, Scientific Computing, Computer Arithmetic, and Validated Numerics, pages 247-256, Cham, 2016. Springer International Publishing. DOI: 10.1007/978-3-319-31769-4_20.

[37] Smith, H.L. Monotone Dynamical Systems: An Introduction to the Theory of Competitive and Cooperative Systems, volume 41. Mathematical Surveys and Monographs, American Mathematical Soc., Providence, 1995. 
[38] Wang, B., Liu, Z., Li, S., Moura, S., and Peng, H. State-of-Charge Estimation for Lithium-Ion Batteries Based on a Nonlinear Fractional Model. IEEE Trans. on Control Systems Technology, 25(1):3-11, 2017. DOI: 10.1109/TCST . 2016. 2557221.

[39] Zou, Ch., Zhang, L., Hu, X., Wang, Z., Wik, T., and Pecht, M. A Review of Fractional-Order Techniques Applied to Lithium-Ion Batteries, Lead-Acid Batteries, and Supercapacitors. Journal of Power Sources, 390:286-296, 2018. DOI: $10.1016 / j$.jpowsour. 2018.04.033. 